\section{Vielfalt des Lernens in einer flexibilisierten Gesellschaft}

Frühjahrstagung der Sektion „Jugendsoziologie" der DGS zusammen mit dem Deutschen Jugendinstitut in München am 22. und 23. März 2004

\section{Renate MÜLleR}

\section{Call for Papers}

Lemprozesse sind heute nichts zuletzt infolge der Mediatisierung und Flexibilisierung sozialer Bezüge durch einen hohen Grad der "Informalisierung" gekennzeichnet. Wie sich unter diesen Bedingungen die Aneignung von Wissen und Kompetenzen vollzieht, ist bisher nur wenig bekannt. Dieses Forschungsdefizit ist auch für Jugendforschung von Bedeutung, denn gerade der Jugendalltag, unabhängig davon, ob dieser Lebensabschnitt als eigenständige Lebensphase oder als Vorbereitung auf das Erwachsenendasein und Berufsleben erachtet wird, kann als höchst lernintensiv bezeichnet werden. Die z. T. technisch induzierten veränderten Lernanforderungen besitzen bislang noch nicht vollständig prognostizierbare Herausforderungen. Es wird zwar häufig versucht, passende Bezeichnungen für einen Wandel der Lernkultur zu finden (z. B. selbstgesteuertes, informelles oder lebenslanges Lernen), doch bleibt wie so häufig die Verbindung von wortgewaltiger Diagnose, theoretisch gehaltvoller Analyse und praktisch-pädagogischer Umsetzung aus.

Mit der Tagung soll auf diesen Umstand reagiert werden. In den Beiträgen sollten die folgenden Punkte fokussiert werden:

a) Die Dynamisierung der Gesellschaft als Auslöser der Informalisierung. Hierbei geht es um die soziologische Deutung der Gegenwartsgesellschaft, vor deren Hintergrund sich sowohl die Jugend, als auch das Lernen verändern.

b) Lernwelten in und neben der Schule. Zu fragen ist, inwieweit die Schule als Ort institutionalisierter Lernprozesse selbst einem Wandel unterworfen ist und welche neuen Lernfelder sich im Jugendalltag ausdifferenzieren (z.B. Technik, Nebenjob, Sport etc.).

c) Grenzen und Probleme des Lernens in der flexibilisierten G esellschaft. Hier spielen u.a. Beobachtungen pädagogischer Provenienz eine Rolle, die möglicher Weise die Überforderung von Jugendlichen oder das Aufscheinen neuer Lem-Ungleichheiten diagnostizieren.
Referatsangebote sollten spätestens bis zum 10. Januar 2004 mit Betreff "Informalisierung" bei Claus J. Tully Deutsches Jugendinstitut, Nockherstr. 2, 81541 München eingehen.

Email: tully@ dji.de

Tel.: 089/ 62306(0) -190 